TEMAS EMERGENTES

\title{
Hernán Santa Cruz: Un diplomático esencial para la Declaración Universal de Derechos Humanos
}

\author{
Hernán Santa Cruz: An essential diplomat \\ for the Universal Declaration of Human Rights
}

Felipe Aravena (D)

American University, Estados Unidos

\begin{abstract}
RESUMEN Hernán Santa Cruz, embajador de Chile ante la Organización de las Naciones Unidas (1947-1952), formó parte del Comité Redactor de la Declaración Universal de Derechos Humanos, el instrumento internacional más emblemático del derecho internacional de los derechos humanos. Como uno de los ocho integrantes del Comité Redactor, tuvo un rol protagónico en los debates y en los trabajos preparatorios. Así, fue un actor clave en aquel momento histórico. Para dimensionar y explicar el aporte de Santa Cruz, es necesario atender a sus especiales características personales y biográficas, entre las que se cuentan su capital cultural y su formación, así como unas circunstancias sociales intelectualmente estimulantes. De esta forma, la visión de Santa Cruz, representativa de una generación latinoamericana, resultaría determinante en su rol como constructor de la Declaración Universal. Este artículo busca explicar las circunstancias que hicieron de Santa Cruz un actor relevante en el Comité de Redacción de la Declaración Universal y dimensionar la magnitud de su contribución a ella.
\end{abstract}

PALABRAS CLAVE Derechos humanos, Declaración Universal de Derechos Humanos, Comité de Redacción, diplomacia, Hernán Santa Cruz.

ABSTRACT Hernan Santa Cruz, Ambassador of Chile to the United Nations (19471952), was part of the Drafting Committee of the Universal Declaration of Human Rights, the most emblematic international instrument of International Human Rights Law. As one of the eight members of the Drafting Committee, he played a leading role in the debates and preparatory work, being a key factor in that historical moment. In order to measure and explain Santa Cruz's contribution, it is necessary to take into account his particular personal and biographical characteristics, including his cultural capital and education, as well as his intellectually stimulating social circumstances. Thus, Santa Cruz's vision, representative of a Latin American generation, would be decisive in his role as a builder of the Universal Declaration. Clave: Derechos Humanos, Declaración 
Universal, Comité Redactor, Diplomacia. This article seeks to explain the circumstances that made Santa Cruz a relevant actor in the Drafting Committee of the Universal Declaration and to measure the magnitude of his contribution.

KEYWORDS Human Rights, Universal Declaration, Drafting Committee, diplomacy, Hernan Santa Cruz.

\section{Introducción}

Hernán Santa Cruz (1906-1999) fue un diplomático latinoamericano que, como embajador de Chile ante la recién creada Organización de las Naciones Unidas, formó parte del Comité Redactor de la Declaración Universal de Derechos Humanos, el instrumento internacional más emblemático del derecho internacional de los derechos humanos. Como uno de los ocho integrantes del Comité Redactor, presidido por la estadounidense Eleanor Roosevelt, tuvo un rol protagónico en los debates y en los trabajos preparatorios que dieron lugar a la aprobación de la Declaración en 1948. Así, fue un actor clave en aquel momento histórico para la diplomacia y los derechos humanos.

Para dimensionar y explicar el aporte de Hernán Santa Cruz, abogado casi sin experiencia diplomática previa que logró plasmar en la Declaración Universal de Derechos Humanos principios e ideas que cobraron enorme relevancia para millones de personas en todo el planeta, es necesario atender a sus muy especiales características personales y biográficas, entre las que se cuentan su capital cultural y su formación, así como unas circunstancias sociales intelectualmente estimulantes que lo llevaron a ser partícipe de una generación de élite del Chile y la América Latina de la posguerra. Así, la visión social, política, jurídica y filosófica de Hernán Santa Cruz, representativa de una generación latinoamericana, resultaría determinante en su rol como constructor de la Declaración Universal de Derechos Humanos.

Este artículo busca explicar, entonces, las circunstancias que hicieron de Santa Cruz un actor relevante en el Comité de Redacción de la Declaración Universal y dimensionar la magnitud de su contribución a la creación de esta pieza esencial del derecho internacional de los derechos humanos.

\section{Contexto familiar y juventud}

Hernán Santa Cruz Barceló nació en 1906 como hijo y nieto de una destacada familia del ámbito público chileno. Su padre fue Joaquín Santa Cruz Ossa, abogado y funcionario del Ministerio de Relaciones Exteriores de Chile, además de alcalde de la comuna de Ñuñoa en la ciudad de Santiago (Ulloa y Medina, 2019: 967). Su abuelo paterno, Joaquín Santa Cruz Vargas, fue senador por el Partido Radical y fundador de 
la Revista Chilena de Historia y Geografía. Su abuelo materno fue José María Barceló, quien ostentó los cargos de senador por Valparaíso, ministro de Justicia (entre 1873 y 1876), decano de la Facultad de Derecho de la Universidad de Chile y presidente de la Corte Suprema (1893).

Como heredero de ese capital político cultural, Hernán pasó su infancia y juventud, como relata en sus memorias (Santa Cruz, 1984: 38-39), devorando lecturas sobre la Revolución Francesa y descubriendo autores panamericanistas en la biblioteca familiar (Ross, 2014: 82). Sin duda, estas lecturas lo impresionaron y estimularon en mayor medida que los textos jurídicos estudiados en la Facultad de Derecho de la Universidad de Chile, cuyas materias no le generaron gran motivación. En cambio, Santa Cruz prefirió adentrarse en los laberintos de la Revolución Rusa e instruirse sobre lo que ocurría en la Europa de entre guerras. Desde entonces, mostraba una clara inclinación por el conocimiento internacionalista que, años más tarde, le sería de mucha utilidad.

Aun así, siguiendo la tradición familiar, dedicó su primera vida profesional a la abogacía, en la que, por sus capacidades y vínculos, rápidamente adquirió respeto y prestigio.

\section{Su amigo Salvador}

Desde fines de la década del treinta, la íntima amistad que trabó con el médico Salvador Allende, en ese entonces ministro de Salud, y que décadas más tarde se convertiría en el primer socialista presidente de Chile (1970-1973), ilustra de buena forma las conversaciones y proyectos de los que Hernán Santa Cruz formó parte en la vida social santiaguina de la primera mitad del siglo XX. Entre diciembre de 1938 y noviembre de 1941, la presidencia de Chile fue detentada por Pedro Aguirre Cerda, militante radical que hizo del lema «gobernar es educar» el símbolo de su período presidencial. Por esos años, Chile se convirtió en país de asilo para muchos latinoamericanos y europeos que encontraron en él un refugio ante la opresión. ${ }^{1}$ Siempre interesado en los asuntos públicos, una vez que culminaba su jornada regular como abogado, Santa Cruz encaminaba sus pasos hacia el Ministerio de Salud para colaborar con Allende en materias como la Ley de Seguro Obrero y la Ley de Accidentes del Trabajo. ${ }^{2}$ En ese mismo período, Allende fue pionero en establecer los vínculos directos entre pobreza y enfermedad en el contexto de la sociedad chilena, conclusión reflejada en su libro La realidad médico-social de Chile, publicado en 1939. Esta tesis resultaría clave para la posterior creación del Servicio Nacional de Salud de Chile y sus importantes logros

1. Mary Zager, "Allende, el amigo», Archivos Salvador Allende, 1988, disponible en https://bit. ly/3HfiEHc.

2. Zager, «Allende, el amigo». 
sanitarios vigentes hasta la actualidad, como las vacunaciones masivas, la erradicación de enfermedades infectocontagiosas y la educación sanitaria, entre otros.

Por aquellos días, Hernán Santa Cruz no solo estrechó profundamente los lazos con Salvador Allende, sino también con exiliados peruanos y venezolanos como el cofundador del Partido Alianza Popular Revolucionaria Americana, Manuel Seoane, el posteriormente vicepresidente aprista de Perú, Luis Alberto Sánchez, y con quien pocos años más tarde sería presidente de Venezuela, Rómulo Betancourt. Con todos ellos, Hernán Santa Cruz se reunía a diario en Santiago para debatir sobre la necesidad de la unidad latinoamericana, para reaccionar a las noticias sobre la guerra que llegaban desde Europa y para analizar ciertas ideas que comenzaban a tomar fuerza para el período de la posguerra, como la creación de las Naciones Unidas.

Los departamentos de Allende y Santa Cruz, vecinos del mismo edificio en la calle Victoria Subercaseaux, en el centro de Santiago de Chile, sirvieron como sede informal para los encuentros entre estos jóvenes latinoamericanos, a los que, en ocasiones, se sumaron personeros como el estadounidense Nelson Rockefeller (Santa Cruz, 1999: 113), quien posteriormente fuera subsecretario de Asuntos Hemisféricos y vicepresidente de Estados Unidos. ${ }^{3}$ En 1950, ya con Santa Cruz como embajador de Chile ante Naciones Unidas, Rockefeller ofrecería un almuerzo en Nueva York en honor del presidente chileno Gabriel González Videla, evento que, por el nivel de las autoridades presentes y la calidez del encuentro, fue considerado un inmenso éxito diplomático para Chile.

Fue también en aquel período cuando se consolidó su amistad con el joven político y abogado Eduardo Frei Montalva, ícono del pensamiento humanista cristiano chileno y quien llegaría a ser presidente de la República entre 1964 y 1970, antecediendo al gobierno de Allende. Junto con las tertulias, eran habituales los paseos y las vacaciones conjuntas entre las familias de estos protagonistas políticos del siglo XX chileno.

Hernán Santa Cruz fue testigo del matrimonio entre Salvador Allende y Hortensia Bussi, y padrino de su hija Carmen Paz. Salvador Allende, en tanto, fue padrino de Adriana, hija de Hernán Santa Cruz y Adriana García de la Huerta. Algunos años después, Allende y Frei viajarían juntos a Nueva York para visitar a Santa Cruz mientras era embajador de Chile ante las Naciones Unidas. Incluso, la primera vez que un órgano principal de Naciones Unidas (exceptuando la sesión de 1948 en París) se reunió fuera de su sede oficial fue el ECOSOC de 1951 en Santiago, iniciativa propuesta por el delegado chileno Eduardo Frei Montalva y aprobada por el Consejo bajo la presidencia de Hernán Santa Cruz (Toro, 2018: 245-261). En sus períodos presidenciales, tanto Frei como Allende encargarían la Representación Permanente de Chile ante las Naciones Unidas y otros Organismos Internacionales con sede en Ginebra a su amigo Hernán.

3. Entre diciembre de 1974 y enero de 1977, durante el gobierno de Gerald Ford. 
En relación con Allende, el propio Hernán Santa Cruz señaló que, durante años, vivieron «prácticamente juntos», y que «muchas veces participaba de sus ideas políticas y otras no». Aunque muy a pesar de Santa Cruz, Allende «nunca fue muy amigo de la literatura», por lo que sus conversaciones giraban en torno a «los sucesos nacionales e internacionales». ${ }^{4}$ Años más tarde, en 1988, Hernán Santa Cruz se referiría al proyecto político de su amigo Salvador, violentamente truncado con el golpe militar del 11 de septiembre de 1973, señalando que:

Tengo la más alta admiración por el proyecto que puso en marcha. Ese proyecto se apoyaba, sobre todo, en mejorar la situación de los sectores más pobres de Chile y de establecer una mayor igualdad. Siempre ha existido en Chile, nunca tanto como hoy, una división más tajante entre los sectores altos y los más empobrecidos. Salvador quería terminar con eso..$^{5}$

En 1999, la senadora Isabel Allende, hija del expresidente, recordaría en el Parlamento chileno que Hernán Santa Cruz, junto con ser uno de los principales asesores de su padre en materias internacionales, colaboró estrechamente en el histórico discurso presidencial pronunciado en la Asamblea General de Naciones Unidas el 4 de diciembre de $1972 .{ }^{6}$ En aquella ocasión, Allende comenzaría su alocución diciendo:

Vengo de Chile, un país pequeño, pero donde hoy cualquier ciudadano es libre de expresarse como mejor prefiera, de irrestricta tolerancia cultural, religiosa e ideológica, donde la discriminación racial no tiene cabida. ${ }^{7}$

Ese mismo año se efectuó, en Santiago de Chile, la Conferencia de la Comisión de las Naciones Unidas para el Comercio y el Desarrollo, que, a instancias y bajo la responsabilidad de Hernán Santa Cruz, se abocó a discutir sobre los principales problemas del comercio y del desarrollo del Tercer Mundo, con la participación de centenares de países y con el respaldo de la comunidad internacional, marcando un hito en la política exterior del gobierno de Allende. ${ }^{8}$

Sin embargo, a pesar de la profunda amistad que lo unía con Frei (demócrata cristiano) y Allende (socialista), las principales simpatías políticas de Hernán Santa Cruz estaban con el Partido Radical. Para ejemplificar cómo en la vida de este diplomático las afinidades personales y políticas se entrecruzaron de manera determinante, cabe remitirse a la importancia que tuvo la amistad surgida con quien también llegaría a ser presidente de Chile, entre 1946 y 1952, el radical Gabriel González Videla.

4. Zager, «Allende, el amigo».

5. Zager, «Allende, el amigo».

6. Diario de sesión: Sesión ordinaria 54, legislatura extraordinaria 339, Senado de Chile, 20 de abril 1999.

7. Salvador Allende, «Discurso en Naciones Unidas», 1972, disponible en https://bit.ly/3eqCRU2.

8. Juan Somavía, «Salvador Allende: El respeto del mundo», El Mostrador, 5 de septiembre de 2020, disponible en https://bit.ly/3FzKVxR. 


\section{Brasil y Gabriel González Videla}

La relación de lealtad y admiración que surgió entre Gabriel González Videla y Hernán Santa Cruz tiene su origen en la ciudad de Río de Janeiro, hasta donde llegó este último en 1944 en calidad de secretario general del Instituto Chileno-Brasileño de Cultura. En ese momento, el embajador de Chile en Brasil era el destacado político Gabriel González Videla. En esos cuatro meses que Santa Cruz vivió en Río de Janeiro como huésped del embajador de Chile, trabajaron juntos en diversas actividades de cooperación diplomática (Santa Cruz 1999: 37), con un ambiente marcado por la constatación de que Brasil no estaba dispuesto a ceder todo el protagonismo y la iniciativa regional a Estados Unidos, (Ross, 2014: 84), por lo que los brasileños desplegaban una activa política exterior hacia América Latina.

En septiembre de 1945, Santa Cruz expresaba parte del núcleo de su ideario filosófico político en una conferencia denominada «Vinculación intelectual entre Brasil y Chile», en la que afirmaba que:

Se necesita ser muy retrógrado, para aceptar como auténtica democracia, una donde las desigualdades económicas no merezcan la menor atención y se niegue a los hombres la identidad de posibilidades económicas o condiciones mínimas de vida (Santa Cruz, 1945).

En esta conferencia, Santa Cruz presentaba su visión político social, caracterizada por una total indivisibilidad e interdependencia entre los derechos civiles, políticos, económicos, sociales y culturales. Esta concepción, fraguada a lo largo de varias décadas de la vida de Santa Cruz, es fundamental para entender la relevancia de sus posteriores aportes en la Comisión Redactora de la Declaración Universal de Derechos Humanos.

En 1946, dos años después de recibir a Santa Cruz en Río de Janeiro, Gabriel González Videla sería electo presidente de la República tras la muerte de Juan Antonio Ríos. En esta campaña presidencial, Santa Cruz participó en la elaboración del Programa de Gobierno de González Videla en materia internacional. Una vez electo presidente, González Videla intentó designar a Hernán Santa Cruz como embajador de Chile en Brasil, sin embargo, el gobierno brasileño no le entregó el beneplácito argumentando que este no tenía la suficiente trayectoria diplomática y política para el cargo. Para el puesto de embajador en Argentina, Chile había designado a Alfonso Quintana, un exvicepresidente de la República, y Brasil esperaba a alguien de similar experiencia (Bernstein, 1984: 101). Fue en ese momento, en diciembre de 1946, cuando el presidente González Videla decidió ofrecer a Hernán Santa Cruz la Representación de Chile ante la naciente Organización de las Naciones Unidas.

González Videla, como exintegrante de la delegación chilena que participó en la Conferencia de San Francisco de 1945, en la que se adoptó la Carta de las Naciones 
Unidas, estaba convencido de que las características personales de Santa Cruz eran ideales para designarlo como el primer representante permanente de Chile ante la naciente organización. Sin embargo, el propio Santa Cruz no parecía estar de acuerdo, y le respondió al presidente que no creía tener las capacidades técnicas para aceptar la propuesta. González Videla insistió, señalándole que el servicio exterior de Chile, al igual que el de la mayoría de los países, no estaba completamente preparado para una diplomacia multilateral como la que se avizoraba en Naciones Unidas, remarcándole su confianza en que sus atributos personales lo harían desempeñar con éxito la tarea. ${ }^{9}$ Este hito marcó un antes y un después en la vida de Hernán Santa Cruz, al punto de que él lo califica como la línea divisoria entre sus «dos existencias»: la primera, que va desde su nacimiento, pasa por su juventud universitaria y se extiende hasta su labor como jurista, y la segunda, que se inicia una vez que comienza su actividad diplomática multilateral a principios de $1947,{ }^{10}$ y que lo llevó a ocupar múltiples cargos de enorme relevancia internacional hasta el final de sus días, en 1999.

\section{Chile, América Latina y el fin de la Segunda Guerra Mundial}

En Chile, en tanto, el Frente Popular, liderado por el Partido Radical, pero con la presencia de los partidos Socialista y Comunista, inició, en 1938, catorce años consecutivos de gobierno, que abarcó las presidencias de Pedro Aguirre Cerda, Juan Antonio Ríos y Gabriel González Videla. Estos triunfos electorales demostraban una simpatía ciudadana mayoritaria por las políticas con acento social que promovía el Frente Popular y el radicalismo, ideas con las que sintonizaba muy bien el pensamiento de Hernán Santa Cruz.

Al finalizar la Segunda Guerra Mundial, Chile y los países latinoamericanos consideraban fundamental insertarse en la naciente Organización de Naciones Unidas, a partir de la premisa de que el nuevo orden mundial abriría una posibilidad real de tener mayor protagonismo político en el ámbito global y la certeza de que no solo Europa y Asia necesitaban el apoyo de la comunidad internacional, sino también aquellos países que habían sufrido indirectamente las consecuencias de la guerra. Si bien la población de América Latina no había participado de los enfrentamientos bélicos ni había sido víctima de asesinatos masivos, la guerra había generado graves consecuencias para la economía de la región, y amplios sectores sufrían desnutrición y muchos otros no tenían acceso a la educación primaria. Esto, sumado a los impac-

9. Para más información, véase Carlos Martínez, «Hernán Santa Cruz Barceló: Un homenaje en la Cepal», Naciones Unidas, 15-18: 16, disponible en https://bit.ly/3FGC79l.

10. Para más información, véase Hernán Santa Cruz, «Discurso para incorporación como miembro número de la Academia Chilena de Ciencias Sociales del Instituto de Chile», 1986. 
tos en el mundo industrial y el consiguiente desempleo, hacían de América Latina una región que merecía la atención y la cooperación internacional.

Por eso, ya instalado en Naciones Unidas, Santa Cruz expresaría que América Latina tenía derecho a exigir que la organización, creada en San Francisco, le prestara ayuda en la difícil tarea de elevar el nivel de vida de sus habitantes (Santa Cruz 1984: 128). Tal convicción del gobierno de Chile, presente también en la generalidad de las delegaciones latinoamericanas, llevó a que, en 1948, se aprobara, por impulso de Hernán Santa Cruz, la Comisión para América Latina y el Caribe (Cepal), organización regional con sede en Santiago de Chile de innegable influencia hasta la actualidad.

\section{La creación de la Cepal y el bloque latinoamericano}

Para Hernán Santa Cruz, la urgencia y la necesidad de la creación de una comisión económica para América Latina era tal, que confesaría que, recién asumido como embajador de Chile ante las Naciones Unidas, «había concebido la idea de proponer el establecimiento de una comisión que se ocupara de los problemas del subdesarrollo socioeconómico de nuestra región», pero que, en el programa del quinto período de sesiones del Consejo Económico y Social de Naciones Unidas que se inauguraría el 19 de julio de 1947, no figuraba ningún punto que permitiera introducir esta propuesta. Por ello, y con los plazos encima, decidió enviar un mensaje urgente a la Cancillería chilena señalando que, «salvo orden en contrario», presentaría dentro de dos días una moción para que se creara una Comisión Económica para América Latina. «No llegó esa orden contraria ni tampoco recibí instrucciones al respecto» (Santa Cruz 1995: 25) relataría Santa Cruz, por lo que oficialmente solicitó al secretario general de Naciones Unidas incluir en el programa del Consejo el punto Proyecto de Resolución tendiente a la creación de una Comisión Económica para América Latina.

La Comisión propuesta por Santa Cruz tendría por objeto estudiar las medidas necesarias para facilitar una acción conjunta destinada a favorecer el progreso económico de América Latina y elevar el nivel de su actividad económica, así como mantener y estrechar los vínculos económicos que los ligan entre sí y también con el resto del mundo (Santa Cruz, 1995: 25). Sus posibilidades de aprobación eran remotas, pues se apartaban de las prioridades establecidas por Naciones Unidas hasta ese momento y contaba con la oposición inicial de al menos Estados Unidos, la Unión Soviética, el Reino Unido, Francia, Canadá y Nueva Zelanda. Además, esta propuesta significaba un cambio de paradigma para la labor de Naciones Unidas en el ámbito del desarrollo (Toro, 2018: 253).

Como en la ONU no había entonces informes o estudios sobre la economía latinoamericana, Hernán Santa Cruz se apoyó en la colaboración de su hermano Alfonso Santa Cruz, quien acababa de terminar estudios de posgrado en la Escuela 
de Economía de Harvard, para presentar el proyecto que mostraba un panorama general de las condiciones socioeconómicas de la región. En aquella intervención, Hernán Santa Cruz aludió a la contribución decisiva, hecha por América Latina, a la victoria aliada al proveerlos de petróleo, cobre y otros minerales, trigo, azúcar, café, algodón, lana y nitratos, a precios congelados a bajos niveles (Santa Cruz, 1995: 26). Junto con los inmediatos apoyos de Cuba y Venezuela, Líbano e India respaldaron la propuesta chilena.

Terminada la presentación de su proyecto y consciente de la reticencia de las grandes potencias, Santa Cruz, en una audaz jugada, propuso la creación de un Comité Especial que estudiara el tema y emitiera una recomendación, el que estaría integrado por Chile, China, Cuba, Estados Unidos, Francia, Líbano, Perú, Reino Unido y Venezuela. Tras múltiples encuentros, debates, borradores y discusiones, el Informe del Comité Especial fue presentado en febrero de 1948 en el sexto período del Consejo Económico y Social de Naciones Unidas. A esas alturas, la propuesta de creación de la Cepal ya sumaba también los apoyos de Francia, el Reino Unido, Países Bajos, Australia, Nueva Zelanda, China y Polonia. Así, finalmente, el 25 de febrero de 1948, el Consejo Económico y Social de Naciones Unidas aprobó con 13 votos a favor, o en contra y 4 abstenciones (Estados Unidos, Canadá, Unión Soviética y Bielorrusia) el nacimiento de la Comisión Económica para América Latina (Santa Cruz, 1995: 32) con sede en Santiago de Chile.

Como se puede apreciar al observar el ambiente internacional de la época, para los dirigentes latinoamericanos resultaba evidente que, en este nuevo orden mundial, se corría el riesgo de profundizar aún más la subordinación y la asimetría de poder con respecto a Estados Unidos (Ross, 2014: 80), por lo que se requería un fino trabajo diplomático que permitiera resguardar los intereses de los países latinoamericanos y su necesidad de desarrollo social y económico, a través de una relación de mutuo beneficio con Estados Unidos y las potencias europeas. Para conseguir aquello, pero también para posicionarse como actores relevantes, era imprescindible adquirir protagonismo político en las nuevas instancias internacionales.

En este escenario de posguerra, cobra especial importancia la Conferencia de Chapultepec celebrada en México, en 1945, y la firma del Tratado Interamericano de Asistencia Recíproca de 1947. En Chapultepec, se intentó avanzar en un plan interamericano de desarrollo y comercio que fuera funcional al progreso de la región, sin embargo, Estados Unidos se mostró más interesado en alcanzar acuerdos que le permitieran acelerar el fin de la guerra, por lo que no hubo compromisos. Sobre este momento que se abría tras el fin de la Segunda Guerra Mundial, Hernán Santa Cruz reflexionó que:

La magnitud del peligro que corríamos, las proporciones enormes del conflicto, con sus necesidades y solicitaciones totales, nos hizo abrir los ojos acerca de la in- 
suficiencia, de la pequeñez y el ningún peso en la balanza mundial, de cada uno de estos países, si actuaban aislados (Santa Cruz, 1945: 8).

Bajo estas consideraciones, durante aquel período, Chile concurrió a la Conferencia de Chapultepec, suscribió la Carta de San Francisco que dio origen a las Naciones Unidas en 1945, impulsó la creación de la Cepal, formó parte del Comité Redactor de la Declaración Universal de Derechos Humanos aprobada en 1948 y se hizo parte del Banco Mundial, el Fondo Monetario Internacional y de la Organización de los Estados Americanos (Ross, 2008: 82). El 1947, el presidente Gabriel González Videla declaraba que la representación chilena en Naciones Unidas debía colaborar leal y desinteresadamente al mantenimiento de la paz», especialmente como «depositarios de cargos que entrañan una fuerte responsabilidad y significan una alta demostración de confianza hacia nuestro país», ${ }^{11}$ respaldando, de esa manera, las actuaciones del embajador de Chile, Hernán Santa Cruz.

Tales eventos, acciones y reflexiones de los gobiernos y autoridades latinoamericanas en general, y de Hernán Santa Cruz en particular, denotan la plena conciencia acerca de la importancia de la cooperación regional y de la necesidad de actuar como bloque en los espacios multilaterales globales. Estos elementos, sumado a la realidad numérica de que, entre los 51 estados fundadores de la ONU en 1945, 20 eran latinoamericanos, ${ }^{12}$ hacían muy atractiva la idea de adoptar posiciones conjuntas que resguardaran adecuadamente los intereses de América Latina. Contar con estos apoyos resultaría fundamental durante la negociación y la aprobación de la Declaración Universal de Derechos Humanos.

\section{La Comisión de Derechos Humanos de Naciones Unidas}

Cuando en 1945 se reunieron delegados de cincuenta estados en la Conferencia de San Francisco, convocada por el estadounidense Franklin Roosevelt, el británico Winston Churchill y el soviético Joseph Stalin, el objetivo de estas tres grandes potencias era alcanzar un acuerdo de seguridad colectiva que evitara la repetición de escenarios como los que dieron lugar a la Segunda Guerra Mundial. Sin embargo, varios de los Estados participantes vieron, en esta reunión, una oportunidad para ampliar el acuerdo internacional a ámbitos como los derechos humanos e incorporar en la agenda asuntos sociales y económicos. En esta posición se ubicaban en general los veinte estados latinoamericanos participantes, ${ }^{13}$ que ya en 1938 , bajo la figura de la

11. Mensaje de su excelencia, el presidente de la República don Gabriel González Videla al Congreso Nacional al inaugurar el período ordinario de sesiones en 1947, Santiago de Chile.

12. Para más información, véase «Estados Miembros fundadores de la ONU», Dag Hammarskjöld Biblioteca, disponible en https://bit.ly/3FyEif8.

13. Argentina, Bolivia, Brasil, Chile, Colombia, Costa Rica, Cuba, República Dominicana, Ecuador, 
Conferencia Interamericana (antecesora de la OEA), había adoptado la «Declaración en Defensa de los Derechos Humanos». ${ }^{14}$

Junto con ello, cabe considerar que, a mediados de la década del cuarenta, predominaban las democracias constitucionales en América Latina, cuyos diseños contemplaban una fuerte protección jurídica de los derechos de los ciudadanos, lo que, en parte, se explica como reacción a la Primera Guerra Mundial, a la Gran Depresión y a los proyectos totalitarios en algunos países de Europa. De hecho, quince de los estados latinoamericanos presentes en San Francisco habían redactado sus Constituciones en la década del treinta y del cuarenta (Morsink, 1999: 130), y en ellas era posible apreciar contenidos identificables con el influyente pensamiento social demócrata y social cristiano del siglo XX (Glendon, 2011: 349).

Por todo ello, no fue tan extraño que, iniciada la Conferencia de San Francisco, los estados latinoamericanos, liderados por Chile, Cuba, México y Panamá, intentaran incorporar un borrador de declaración de derechos humanos a la Carta de la ONU. Ese intento fracasó por la indiferencia e incluso por la hostilidad de las grandes potencias frente a la iniciativa, pero la publicación internacional de fotografías de los campos de concentración, en Europa, fue un factor decisivo para que el contenido de derechos humanos estuviera presente en la Carta fundacional de Naciones Unidas, incluida la provisión de constituir una comisión de derechos humanos (Glendon, 2011: 342). Una vez instalada la Organización de Naciones Unidas y su Consejo Económico y Social (ECOSOC), nació la Comisión de Derechos Humanos, la que quedó conformada por dieciocho estados, incluyendo tres de América Latina: Chile, Panamá y Uruguay. ${ }^{15}$

\section{El borrador latinoamericano de la Declaración Universal de Derechos Humanos}

La primera tarea de la Comisión fue encargar la elaboración de un borrador de proyecto de Declaración al director de Derechos Humanos de la ONU, el abogado canadiense John Humphrey. La labor de Humphrey sería muy importante, pues se le hizo responsable de recopilar los documentos de distintas tradiciones jurídicas y filosóficas que servirían como base para ir dando forma, con el paso de las negociaciones, al texto definitivo de la Declaración Universal de Derechos Humanos. Pensadores como Jacques Maritain, Aldous Huxley, Herbert George Wells o Mahatma Gandhi,

El Salvador, Guatemala, Haití, Honduras, México, Nicaragua, Panamá, Paraguay, Perú, Uruguay y Venezuela.

14. Carnegie Endowment for International Peace, International Conference of American States, 19331940 (1943), p. 246.

15. Los otros quince estados fueron Australia, Bélgica, Bielorrusia, China, Egipto, Estados Unidos, Francia, India, Irán, Líbano, Filipinas, Reino Unido, Ucrania, Unión Soviética y Yugoslavia. 
entre muchos otros, aportaron a Humphrey en esta etapa previa (Glendon, 2011: 343). Teniendo muchísimo material a la vista, John Humphrey decidió que sus modelos principales para la elaboración del primer borrador serían las propuestas aportadas por Panamá y Chile.

La propuesta de Panamá consistía en el mismo proyecto presentado sin éxito en la Conferencia de San Francisco de 1945, el que se basaba en el trabajo realizado por el Instituto de Derecho Americano entre 1942 y 1943 con participación de un comité multinacional y que fue denominado como «Declaración de Derechos Humanos Esenciales». La propuesta de Chile, en tanto, se trató de una versión preliminar de la Declaración Americana de los Derechos y Deberes del Hombre, que se venía trabajando desde 1945 en el Comité Jurídico Interamericano de la OEA y que se aprobaría posteriormente en Bogotá, en abril de 1948 (Morsink, 1999: 131). Es decir, al momento de presentar el borrador en el Comité de Derechos Humanos, Chile negociaba paralelamente la Declaración Americana de los Derechos y Deberes del Hombre en la OEA, y la Declaración Universal de Derechos Humanos en la ONU.

Con estos antecedentes, y basándose fundamentalmente en las propuestas panameña y chilena, John Humphrey incorporó, en el primer borrador, además de los clásicos derechos civiles y políticos, los derechos sociales, económicos y culturales presentes en los textos latinoamericanos. Una de las principales virtudes que estos ofrecían era que lograban compatibilizar las distintas tradiciones políticas y filosóficas presentes en Naciones Unidas, constituyendo una opción sincrética entre la concepción jurídica angloamericana y la europea continental. Mientras la tradición angloamericana destacaba la libertad individual y el derecho de propiedad, el derecho continental europeo ponía el acento en la igualdad y en la fraternidad, conceptualizando al Estado como garante y protector. Las propuestas de Panamá y Chile, en tanto, lograban conciliar ambas filosofías de manera armónica, pues los estados independientes latinoamericanos, surgidos en el siglo XIX, habían erigido sus sistemas jurídicos tomando como referencia los documentos fundacionales de Estados Unidos y los principios emanados de la Revolución Francesa. Así, el constitucionalismo latinoamericano, bien reflejado en la Declaración Americana de los Derechos y Deberes del Hombre, resultaba muy útil para comenzar a discutir un proyecto de Declaración Universal de los Derechos Humanos.

Las evidentes similitudes apreciables entre la Declaración Americana de los Derechos y Deberes del Hombre y la Declaración Universal de Derechos Humanos, ambas aprobadas en 1948, quedan de manifiesto desde el inicio. Mientras el preámbulo de la Declaración Americana reza que «todos los hombres nacen libres e iguales en dignidad y derechos y, dotados como están por naturaleza de razón y conciencia, deben conducirse fraternalmente los unos con los otros», el artículo 1 de la Declaración Universal establece que «todos los seres humanos nacen libres e iguales en dignidad y derechos y, dotados como están de razón y conciencia, deben comportarse frater- 
nalmente los unos con los otros». Así, la decisión de John Humphrey, de basar su primer borrador de Declaración Universal en las propuestas de Chile y Panamá, tuvo un impacto decisivo en el resultado final, pues sentó las bases para una negociación que condujo al reconocimiento universal de derechos propios del constitucionalismo latinoamericano de la época, como, por ejemplo, el derecho a la salud, al trabajo, a la seguridad social, y a los beneficios de la cultura y la ciencia, todos ellos reconocidos en términos muy similares en ambas declaraciones.

Específicamente, en relación con los derechos económicos, sociales y culturales, y a diferencia de la idea bastante extendida de que fue el bloque soviético quien promovió su presencia en la Declaración Universal, fue el representante de Chile, Hernán Santa Cruz, quien asumió el liderazgo con respecto a la incorporación de este contenido en la Comisión de Derechos Humanos y en el Comité Redactor de la Declaración (Glendon, 2011: 348). Tanto es así, que en la Primera Sesión de Redacción, cuando aún no se sabía con certeza si los derechos sociales, económicos y culturales se incluirían en la Declaración, o cuántos de ellos podrían ser aceptados, Santa Cruz tomó la palabra para expresar que «la declaración, por breve que sea, debería incluir todos los puntos que la humanidad espera sean incluidos en este período de nuestra historia», agregando que «el reconocimiento de estos derechos (económicos, sociales y culturales) haría imposible el regreso del fascismo» (Morsink, 1999: 89)

Así entonces, una vez que John Humphrey incluyera la mayoría de los derechos sociales, económicos y culturales en el primer borrador, ningún otro delegado estuvo más atento de defender su presencia en la Declaración que Hernán Santa Cruz (Morsink, 1999: 89).

\section{Santa Cruz en el Comité de Redacción}

Como se lo había advertido al presidente Gabriel González Videla, Santa Cruz no tenía mayor experiencia diplomática al asumir la Representación de Chile ante las Naciones Unidas. Por ello, los días previos a las primeras sesiones y consciente de que estaría huérfano de todo consejo (Santa Cruz, 1984: 98), Hernán Santa Cruz se preparó leyendo todo tipo de informes, actas y proyectos. La angustia y la ansiedad que reconoce haber sentido esos días, se sumó al asombro que le produjo la calidad intelectual de los representantes de las delegaciones que iba conociendo en Naciones Unidas. Entre ellos destacaban figuras de la talla de Eleanor Roosevelt (Estados Unidos), Charles Malik (Líbano), P.C. Chang (China) y René Cassin (Francia), entre otros. Frente a ellos, escuchó, observó y preguntó mucho, transformándose rápidamente, por su liderazgo y capacidad, en un actor muy relevante en la Organización. La inicialmente improbable creación de la Cepal y su pronta elección como presidente del Consejo Económico y Social de la ONU, en 1950, así lo demuestran. También llegaría a ser presidente de la Primera Conferencia Mundial de Asistencia Técnica 
(1950), presidente y relator de la Comisión sobre la situación racial en Sudáfrica (1953-1955), subdirector general de la FAO (1959), presidente de la Subcomisión de Prevención de la Discriminación y Protección de las Minorías (1964-1965), y presidente del Primer Congreso Mundial de Reforma Agraria (1966), entre otras tantas responsabilidades asumidas que reflejan la dimensión alcanzada por Hernán Santa Cruz en el ámbito internacional (Rojas, 2018: 398).

Cuando a inicios de 1947 se reunió por primera vez la Comisión de Derechos Humanos, su presidenta, Eleanor Roosevelt, inmediatamente comprendió que elaborar un proyecto de Declaración entre dieciocho integrantes sería una tarea extremadamente compleja. Por ello, luego de intentar trabajar con un pequeño Comité de tres integrantes, que demostró ser poco representativo, se decidió generar un Comité de Redacción compuesto por ocho miembros. Este sería el grupo que, basado en la propuesta de Humphrey, desarrollaría el trabajo más arduo para redactar la definitiva Declaración Universal de Derechos Humanos.

El Comité de Redacción de la Declaración Universal de Derechos Humanos quedó conformado por los representantes de Australia, Chile, China, Estados Unidos, Francia, Líbano, Reino Unido y la Unión Soviética, quienes se reunieron por primera vez en tal condición en junio de 1947. En aquella ocasión, los ocho miembros pudieron conocer y discutir el primer borrador de Declaración Universal de Derechos Humanos, que incluía derechos civiles y políticos como la protección a la vida, la libertad y la propiedad, la libertad de expresión, religión y reunión, y también derechos económicos y sociales como el derecho al trabajo, a la educación y a la seguridad social. Sobre este borrador, se encargó al francés René Cassin realizar una edición que comenzó a dar forma a la Declaración, con Preámbulo y artículos ordenados de manera «lógica» (Glendon, 2011: 114). El trabajo de Cassin tuvo el mérito histórico y jurídico de mantener los componentes civiles, políticos, económicos, sociales y culturales de manera coherente y declarando su universalidad. Con ello, se transformaría a cada individuo en sujeto de derecho internacional con respecto a estos derechos. Esto, que actualmente podría parecer tan obvio, tenía un enorme significado político, jurídico, filosófico y moral en aquel momento, especialmente si se considera que recién finalizaba la atrocidad humanitaria de la Segunda Guerra Mundial. Sin embargo, estas primeras propuestas aún debían ser sometidas a la discusión del Comité de Redacción, la Comisión de Derechos Humanos y el Consejo Económico y Social, antes de llegar a la Asamblea General de las Naciones Unidas. Además, actores esenciales como Estados Unidos querían que la declaración fuera lo más «corta y vacía» posible, y a la Unión Soviética directamente le desagradaba la idea de adoptarla en cualquiera de sus formas (Glendon, 2011: 142).

En ese escenario, durante los trabajos preparatorios del Comité de Redacción, en 1947, el Embajador Santa Cruz hizo uso de la palabra para recordar que: 
En San Francisco, cuando los horrores de la guerra y del totalitarismo estaban aún frescos en la memoria del mundo, se reconoció que, para eliminar las causas de la guerra, había que limitar la soberanía de los Estados por consideraciones de solidaridad y cooperación internacional y elevar el nivel económico de los pueblos del mundo (Schabas, 2013: 1.667).

Así, agregó que:

Los derechos económicos y sociales deben encontrar su lugar en la Declaración; el derecho al trabajo, el derecho a un salario equitativo, el derecho a la salud, a la educación y a la seguridad social, y a los beneficios de la cultura y del progreso científico no deben ser omitidos. La delegación chilena no puede apoyar una Declaración que no recoja estos principios» (Schabas, 2013: 1.667).

Este tipo de intervenciones generaron que Santa Cruz rápidamente se transformara en un actor determinante en los debates de derechos humanos.

\section{Defensor de los derechos económicos, sociales y culturales}

Los temas que mayor discusión generarían en el Comité de Redacción fueron los vinculados a los derechos económicos y sociales. Por ejemplo, con respecto al derecho al trabajo, Estados Unidos consideraba que todos los seres humanos debían tener meramente la «oportunidad» de acceder a los ingresos necesarios para vivir, mientras Francia y Chile opinaban que debía reconocerse el derecho a «desempeñar un trabajo socialmente útil». En este punto, Santa Cruz recordó a Estados Unidos que, en un discurso pronunciado en 1943, el presidente Roosevelt se refirió a la necesidad de una nueva Carta de Derechos de los Estados Unidos, que estableciera el derecho al trabajo útil y remunerado, añadiendo que este derecho había sido adoptado por unanimidad en las reuniones de la Organización Internacional del Trabajo y en las Conferencias de Seguridad Social (Schabas, 2013: 872), y que, por lo tanto, el trabajo debía ser recompensado de manera que satisfaciera las necesidades del individuo (Schabas, 2013: 739). En ese mismo debate, Santa Cruz admitió que «la realización del derecho al trabajo en todos los países es una aspiración para el futuro, pero si la Declaración se ajusta solo a las condiciones existentes, no logrará un propósito muy útil» (Schabas, 2013: 837). Con estas palabras, Hernán Santa Cruz revelaba su mirada profundamente progresista sobre el sentido del Derecho Internacional de los Derechos Humanos.

En relación con el rol del Estado en la economía, el embajador Santa Cruz afirmaría que la postura de Chile era que:

El Estado debe intervenir en la orientación de la economía interna de un país, suministrando lo que la iniciativa privada no es capaz de dar y procurando que la producción y distribución de los bienes se ajuste al bienestar general de la población (Schabas, 2013: 245). 
Frente a los cuestionamientos de otros delegados, que replicaron en el sentido de que ese criterio constituía una limitación de la libertad y la democracia, Hernán Santa Cruz respondió que la postura chilena buscaba transformar la democracia política del siglo XIX en una democracia económica (Schabas, 2013: 246).

En el mismo sentido, con respecto al derecho de propiedad, Santa Cruz señalaría, en el Comité de Redacción, que la propuesta chilena consistía en «reconocer el derecho de propiedad y establecer el derecho del Estado a cooperar con los individuos para que tengan un mínimo de propiedad privada acorde con sus necesidades y para que puedan mantener su dignidad», como se había establecido en la Declaración Americana de los Derechos y Deberes del Hombre. El gobierno chileno, dijo Santa Cruz, «no solo quiere que no se limite la propiedad, sino que quiere que se establezca la función social de la propiedad» (Schabas, 2013:808), sugiriendo, entonces, que se necesitaba una fórmula que estableciera el derecho a la propiedad privada, y que reconociera también los derechos de la comunidad con respecto a toda la propiedad. Sobre el punto, afirmaría la importancia de que nadie sea privado de su propiedad sino por causa de utilidad pública y con justa indemnización. El resultado de esta discusión se puede apreciar en el artículo 17.1 de la Declaración Universal de Derechos Humanos, que reconoció que «toda persona tiene derecho a la propiedad, individual y colectivamente», ${ }^{16} \mathrm{y}$ en el 17.2, que establece que «nadie será privado arbitrariamente de su propiedad».

Con respecto al derecho a la seguridad social establecido en el artículo 22 de la Declaración Universal, Santa Cruz declaró, en noviembre de 1948, que «es uno de los más importantes de la declaración en la medida en que hace especial hincapié en el derecho del individuo a un nivel de vida adecuado para él y su familia y garantiza su seguridad social» (Schabas, 2013: 2655). Este mismo artículo constituye también una base fundamental de la Cooperación Internacional cuando señala que:

Toda persona, como miembro de la sociedad, tiene derecho a la seguridad social, y a obtener, mediante el esfuerzo nacional y la cooperación internacional, habida cuenta de la organización y los recursos de cada Estado, la satisfacción de los derechos económicos, sociales y culturales, indispensables a su dignidad y al libre desarrollo de su personalidad.

Considerando la fuerte identificación política de Hernán Santa Cruz con el ideario de los gobiernos radicales en Chile, uno de cuyos principales estandartes fue el fortalecimiento de la educación pública, cabe rescatar su intervención en el debate con respecto al derecho a la educación y su rol clave para que la Declaración Universal reconociera la educación primaria como obligatoria. El francés René Cassin trajo

16. Para más información, véase el artículo 17 de la Declaración Universal de Derechos Humanos del 10 de diciembre de 1948, Naciones Unidas, disponible en https://bit.ly/32EgsQm. 
el derecho a la educación al debate del Comité de Redacción y luego a la Comisión de Derechos Humanos. En ambas ocasiones, encontró en Hernán Santa Cruz su principal aliado para apoyar las disposiciones que reconocían el derecho a la educación y a la gratuidad en la educación primaria. Incluso, Santa Cruz afirmó que apoyaría todas las enmiendas que significaran también «la gratuidad de la enseñanza secundaria e incluso de la universitaria», ya que este tipo de enseñanza era gratuita en Chile en aquella época. Sin embargo, el embajador chileno fue muy insistente en que a estas propuestas debía agregarse la obligatoriedad de la educación primaria (Schabas, 2013: 878), lo que fue secundado por la delegación de Australia (Schabas, 2013: 880). Esta idea fue finalmente recogida en el artículo 26.1 de la Declaración Universal de Derechos Humanos, que reza "la instrucción elemental será obligatoria». ${ }^{17}$

Es de toda justicia destacar también el rol de Hernán Santa Cruz en el reconocimiento del derecho a la ciencia en la Declaración Universal de Derechos Humanos, del que encontramos antecedentes en el célebre Discurso de las Cuatro Libertades pronunciado por el presidente Franklin D. Roosevelt en enero de 1941 ante el Congreso de los Estados Unidos. El derecho a la ciencia estuvo presente en el primer borrador presentado al Comité de Redacción por John Humphrey, basado en la Declaración Americana y también en el artículo 38 de la Carta de la OEA (Mancisidor, 2017: 213). En esta versión inicial, el derecho a la ciencia se contemplaba como «el derecho a participar en los beneficios de la ciencia» para transformarse, con el paso de las discusiones, en «el derecho a participar en el avance científico» y, finalmente, por propuesta cubana apoyada por Francia y Chile, en «el derecho a participar en los beneficios que resulten del avance científico». En este debate, Santa Cruz defendió la idea de que las invenciones científicas deben ponerse a disposición de todos los individuos (Schabas, 2013: 878), afirmando también que las invenciones científicas deben pertenecer a la sociedad y ser disfrutadas por todos (Schabas, 2013: 879). Ciertamente con orgullo, el embajador Santa Cruz recordó, durante el debate de la propuesta cubana, que la delegación chilena fue la primera en proponer que la Declaración Universal mencionara el derecho de toda persona a participar en los beneficios del progreso científico, fórmula que reafirmaba, en términos más amplios, los principios adoptados por la Declaración Americana.

\section{Los derechos civiles y políticos}

En todo caso, el protagonismo de Santa Cruz no solo se limitó a los asuntos vinculados a los derechos económicos, sociales y culturales. Por ejemplo, claves fueron sus intervenciones en favor del derecho a la nacionalidad, a no ser privado arbitra-

17. Para más información, véase el artículo 26 de la Declaración Universal de Derechos Humanos del 10 de diciembre de 1948, Naciones Unidas, disponible en https://bit.ly/32EgsQm. 
riamente de ella y del derecho a cambiar de nacionalidad, recogidos en el artículo 15 de la Declaración Universal. De hecho, la propuesta chilena de que «toda persona tiene derecho a una nacionalidad», fue recogida textualmente en el documento final (Schabas, 2013: 823). También apoyó decididamente el concepto fundamental de que «nadie podrá ser arbitrariamente detenido», del artículo 9, y la necesidad de una declaración explícita del derecho de amparo, como la que finalmente recogió el artículo 8 a propuesta de México. La delegación chilena había instado a que se estableciera expresamente que una persona solo podía ser detenida de conformidad con las leyes vigentes, que debía ser juzgada rápidamente y que debía ser liberada de inmediato si era detenida injustamente (Schabas, 2013: 2.355). De igual manera, Santa Cruz respaldó firmemente la enmienda británica y francesa que establecía la igualdad de derechos del hombre y de la mujer en cuanto al matrimonio, durante el matrimonio y en caso de disolución de este, recogidos en el artículo 16 de la Declaración Universal de Derechos Humanos.

Hernán Santa Cruz también lideró la inclusión del derecho al asilo de manera amplia, en oposición a la propuesta más restrictiva de la Unión Soviética, que postulaba que el derecho de asilo solamente debía concederse a las personas perseguidas por haber defendido los intereses de la democracia o por su actividad científica o por su participación en la lucha por la liberación nacional (Schabas, 2013: 2.425). Santa Cruz replicó que, con la propuesta soviética, el ámbito de aplicación del artículo 14 sería singularmente restrictivo, ya que, por ejemplo, no se consideraba a las víctimas de persecuciones religiosas o raciales. Finalmente, el artículo 14 de la Declaración recogió una fórmula más amplia que señala que «en caso de persecución, toda persona tiene derecho a buscar asilo, y a disfrutar de él, en cualquier país». Si bien esta fórmula no satisfizo totalmente las expectativas chilenas que, en línea con la Declaración Americana, postulaban que «toda persona tiene el derecho de buscar y recibir asilo», ${ }^{18}$ Santa Cruz adhirió a ella por considerarla una mejor opción que la soviética.

Santa Cruz sostuvo variados duelos explícitos y velados con los delegados del bloque soviético en lo referente a derechos civiles y políticos. A propósito de las libertades, en la Sesión del ECOSOC, de 26 de agosto de 1948, argumentaría ardorosamente que:

La libertad, uno de los mayores tesoros del hombre, corre ahora un gran peligro debido a la herencia dejada por la guerra y a la ideología que sostiene que, en la evolución hacia la libertad definitiva, debe haber primero una etapa de recorte de las libertades individuales. Sin embargo, al igual que en el pasado la libertad ha sobrevivido a las peores pruebas de la historia, en el futuro sobrevivirá a cualquier nuevo intento totalitario de destruirla (Schabas, 2013: 2.010).

18. Para más información, véase el artículo 27 de la Declaración Americana de los Derechos y Deberes del Hombre, aprobada en la Novena Conferencia Internacional Americana de 1948, disponible en https://bit.ly/3mzz772. 
Esta intervención fue inmediatamente protestada por el delegado soviético Alexei Pavlov.

\section{La «Ley Maldita», «Los Mentirosos» y los poetas}

A propósito de los debates con la delegación soviética, cabe mencionar que, como embajador nombrado por el presidente Gabriel González Videla, la relación de Santa Cruz con el bloque comunista de Naciones Unidas estuvo determinada también por los acontecimientos internos de la política chilena. En el marco de los alineamientos y distanciamientos globales que surgían con la Guerra Fría, aunque no solo por ello, el presidente Gabriel González Videla promulgó, en septiembre de 1948, la «Ley de Defensa Permanente de la Democracia», conocida popularmente en Chile como Ley Maldita, que proscribió la participación política del Partido Comunista de Chile, partido inicialmente miembro de la coalición de gobierno. Esta ley significó la eliminación de los militantes comunistas del registro electoral, la pérdida de sus cargos electoralmente conseguidos, inhabilidades, sanciones, persecución política y exilio. Emplazado por esta violenta política adoptada por el gobierno de Chile, que implicaba también un fuerte ataque al sindicalismo chileno, Santa Cruz respondió en Naciones Unidas que la ley había sido aprobada cumpliendo todos los requisitos que establecía el ordenamiento jurídico nacional (Ulloa y Medina, 2019: 978), y que su gobierno estimaba que era necesario contar con disposiciones que defendieran pronta y eficazmente el régimen institucional y la economía nacional, puestos en peligro por una conspiración inspirada en intereses ideológicos, políticos y económicos de una potencia extranjera (Ulloa y Medina, 2019: 978). Esta contradictoria declaración en defensa de su gobierno, por medio de la cual justificaba la persecución política de los comunistas chilenos y la desarticulación sindical, traería consecuencias inesperadas para Hernán Santa Cruz.

Esto, por cuanto la Ley de Defensa Permanente de la Democracia no solo implicó un costo político enorme para el Partido Radical chileno, que luego de González Videla no volvió a ver a un militante suyo ocupar la presidencia de la República, sino que incluso le significó, a los más insignes diplomáticos chilenos de la época, una oprobiosa reprobación inmortalizada por el militante comunista y Premio Nobel de Literatura, Pablo Neruda. El poeta chileno dedicó algunos despectivos versos a Hernán Santa Cruz y sus colegas en el «Canto XIII» de su popular obra Canto General, bajo el título de «IX. Los Mentirosos»:

Hoy se llaman Gajardo, Manuel Trucco

Hernán Santa Cruz, Enrique Berstein, Germán Vergara, los que - previo pagodicen hablar, oh Patria, en tu sagrado nombre y pretenden defenderte hundiendo 
tu herencia de león en la basura. Enanos amasados como píldoras en la botica del traidor, ratones del presupuesto, mínimos mentirosos, cicateros de nuestra fuerza, pobres mercenarios de manos extendidas y lenguas de conejos calumniosos. No son mi patria, lo declaro a quien me quiera oír en estas tierras, no son el hombre grande del salitre, no son la sal del pueblo transparente, no son las lentas manos que construyen el monumento de la agricultura, no son, no existen, mienten y razonan para seguir, sin existir, cobrando.

Distinta sería la relación surgida entre Hernán Santa Cruz y la primera chilena Premio Nobel de Literatura, Gabriela Mistral (Ulloa y Medina, 2019: 972). Santa Cruz sentía una profunda admiración intelectual por la poeta y los unían similares simpatías políticas. Revelador es el artículo que le dedica en la Revista Zig-Zag en 1945 tras visitarla en su casa en Río de Janeiro, en el que confiesa que:

Constituye una verdadera fiesta para el espíritu escuchar conversar a Gabriela. Su poderoso intelecto, alumbrado por una gran bondad y por una cultura superior adquirida en los libros y también en su infatigable correr por el mundo con los ojos bien abiertos - irradiaba fuerza, superioridad moral, energía pensante.

Sobre esa visita a Gabriela Mistral, Santa Cruz recuerda que la poeta le explicó lo que ella entendía como "patriotismo activo», que consistía en su manera de hacer ver los vicios y defectos nacionales, lo que le generaba mucha incomprensión en Chile. En este punto, Gabriela Mistral lamenta que, en Chile, se esté desarrollando «un falso y absurdo orgullo nacional, que se apresura a encontrar lo chileno superior a lo de los demás países». A esta reflexión, Hernán Santa Cruz responde que esta apreciación «se debe, seguramente, a que los compatriotas que ella frecuentaba en el extranjero estaban en pleno período de euforia prepotente al sentirse de pronto liberados, aligerados, del aplastamiento que nos producen la pequeñez, la chismografía y las tejas santiaguinas». Sin duda, Mistral y Santa Cruz, cada uno con su estilo y personalidad propia, compartían una mirada inconformista sobre la realidad social chilena. 


\section{La batalla del Preámbulo y «la guía espiritual para la humanidad»}

Una batalla a la que dedicó largas jornadas Hernán Santa Cruz fue a aquella sostenida en Naciones Unidas acerca del contenido que debía tener el Preámbulo de la Declaración Universal de Derechos Humanos. Además de su intransigencia con respecto a que el Preámbulo debía hacer mención expresa a los derechos económicos, sociales y culturales (Schabas, 2013: 825) - y también ser instalados entre los primeros artículos- para que se reflejara adecuadamente la importancia de estos y «estar en armonía con la realidad del mundo actual» (Schabas, 2013: 848), también defendió ardorosamente que el texto, en su conjunto, tuviera un carácter laico y no discriminatorio hacia ninguna religión en particular. El 30 de noviembre de 1948, ad portas de la aprobación de la Declaración Universal y con el preámbulo prácticamente culminado, el embajador Santa Cruz calificaría, por fin, esta parte del texto como «satisfactorio y ajustado al contenido de la declaración». Con respecto a las últimas enmiendas presentadas, señalaría que:

La de Ecuador no menciona los derechos económicos y sociales, que, en cambio, están incluidos en la enmienda de la URSS. Podría ser un buen plan para el Comité basarse en esta última enmienda para mejorar el texto del preámbulo (Schabas, 2013: 2.906).

El representante chileno, en aquella ocasión, subrayó también la dificultad de adoptar una propuesta de enmienda holandesa referida al origen divino del ser humano. Santa Cruz, entonces, recordó que:

Chile, donde el grueso de la nación es sinceramente católico, no menciona la Providencia en su Constitución, por respeto a las convicciones de una importante minoría. Por razones similares, Chile no apoyará la enmienda holandesa (Schabas, 2013: 2.906).

Fue quizás por este tipo de posicionamientos que John Humphrey, quien reconocía mucha afinidad con el embajador chileno (Morsink, 1999: 131), diría que Hernán Santa Cruz ejercía una influencia considerable sobre las delegaciones que representaban a los países en desarrollo, por quienes argumentaba con gran energía; una práctica que con frecuencia lo enfrentó con las potencias industriales de Occidente (Humphrey, 1984: 37).

Junto con ello, es muy destacable que Santa Cruz parecía haber adquirido temprana conciencia de la trascendencia de lo que se estaba negociando. Recién instalado el Comité de Redacción, quedaría en actas que el embajador chileno postularía que el Comité debe elaborar una Carta de Derechos Humanos dándole no solo forma jurídica, sino «un verdadero contenido humano», expresando su convencimiento «de que la Carta Internacional de Derechos Humanos no debe ser solo un proyecto de 
ley, sino una verdadera guía espiritual para la humanidad que enumere los derechos del hombre que deben ser respetados en todas partes» (Schabas, 2013: 719).

Más avanzadas las negociaciones, diría que, «aunque la Declaración no tenga la fuerza de una obligación legal, tendrá, en el futuro, una fuerza moral. La Declaración dista mucho de ser perfecta, pero contiene la esencia de los ideales de las Naciones Unidas».

Para Santa Cruz, la Declaración no era «estéril ni vaga y sus principios inspirarían algún día a los hombres a respetarse a sí mismos» (Schabas, 2013: 2.010). Por lo mismo, sostenía que, jurídicamente:

Los Estados miembros, al suscribir el artículo 55 de la Carta de Naciones Unidas, se han comprometido formalmente a respetar los derechos humanos. Se trata de una obligación legal para ellos; los que no la cumplen se exponen a las sanciones previstas en la Carta o, en todo caso, a la censura moral del mundo. Al firmar la Carta, los Estados aceptaron limitar voluntariamente su soberanía nacional. La Carta no contiene una enumeración de los derechos humanos; impone a los Estados miembros la obligación de respetarlos (Schabas, 2013: 2.786)

En esta línea de razonamiento, Santa Cruz era partidario de reconocer el derecho de los individuos a presentar peticiones a las Naciones Unidas, ya que «al suscribir la Carta, los Estados han aceptado la incorporación de los derechos humanos al derecho internacional y han abandonado voluntariamente parte de su soberanía». Por lo tanto, consideraba que debía «existir una autoridad internacional que examinara las peticiones relativas a los derechos humanos.» (Schabas, 2013: 2.820).

\section{Un liderazgo pragmático para la aprobación de la Declaración Universal}

Probablemente, por esa plena conciencia que tenía Santa Cruz de la centralidad de lo que se estaba discutiendo y de la oportunidad histórica que tenían los estados para aprobar un documento como la Declaración Universal, es que fue muy hábil y pragmático a la hora de alinear las posiciones latinoamericanas en procura del objetivo mayor. Durante el segundo semestre de 1948, con la Declaración Universal muy avanzada, un grupo de países de América Latina decidió plantear la idea de encargar al Comité de Redacción la revisión, línea por línea, de las concordancias entre la Declaración Americana de los Derechos y Deberes del Hombre (Bogotá) y la Declaración Universal de Derechos Humanos. Como esto no fue aprobado, algunos estados latinoamericanos se organizaron para forzar el ajuste de ambas declaraciones en los debates venideros. Sin embargo, esta tarea ponía en riesgo la posibilidad de llegar con el texto final acordado a la Tercera Sesión de la Asamblea General, fijada para diciembre de 1948. Esto, por cuanto la iniciativa podría ser aprovechada por el bloque soviético para dilatar las discusiones hasta diluir los acuerdos ya alcanzados. 
El momento sería bautizado por el canadiense John Humphrey como la Amenaza de Bogotá (Glendon, 2011: 212).

Si bien el objetivo de hacer coincidir de manera casi exacta los textos era apreciado por Santa Cruz, el delegado chileno se daba cuenta de que esa tarea podía hacer fracasar el trabajo completo. Por ello, durante este debate, pidió a los representantes latinoamericanos que recordaran las dificultades enfrentadas para alcanzar un documento aplicable a distintos sistemas económicos, sociales, jurídicos y culturales. Así, el embajador chileno expuso que los estados no debían olvidar que:

El proyecto de declaración fue el resultado de dos años de laborioso esfuerzo por parte de varios órganos de las Naciones Unidas para alcanzar un compromiso, aceptable al menos para la mayoría, de todos esos puntos de vista conflictivos. El texto de la declaración de Bogotá no se ha pasado por alto. Aunque no hay una concordancia total entre él y el proyecto de Declaración, se ha estudiado a fondo y se han aceptado muchos de sus artículos (Schabas, 2013: 2.058).

Santa Cruz tampoco creía conveniente formar una subcomisión para realizar una concordancia, como sugería Cuba. El representante chileno, coincidiendo con la presidenta de la Comisión de Derechos Humanos, Eleanor Roosevelt, consideraba que el proyecto de declaración no era perfecto ni podía serlo, pero que resultaba esencial su aprobación en la Sesión de diciembre de 1948. La Guerra Fría ya comenzaba a mostrar sus mayores complejidades y no era razonable confiar en que los escenarios serían mejores en el futuro próximo.

En otra ocasión, a fines de septiembre de 1948, se debía escoger al delegado que presidiría el Grupo Revisor de la Declaración Universal. Buena parte de los delegados latinoamericanos, que en conjunto representaban más de un tercio de los votos en Naciones Unidas, adhería a la opción de elegir al haitiano Emile Saint-Lot. El representante chileno admiraba la oratoria de Saint Lot y lo apodaba «el Danton Negro» (Glendon, 2011: 208). No obstante, le preocupaba de sobremanera que su falta de experiencia dirigiendo debates en Naciones Unidas pudiera entrampar la aprobación de la Declaración Universal, pues ya había suficientes interesados, especialmente en el bloque soviético, en postergar el acuerdo. Por ello, Santa Cruz dedicó aquellos días a convencer a sus colegas latinoamericanos acerca de la conveniencia de elegir al experimentado Charles Malik, representante del Líbano, como presidente del Grupo Revisor y al haitiano Saint-Lot como relator. Finalmente, la conducción de Malik resultó clave para la aprobación del borrador de la Declaración Universal y el propio embajador chileno recordaría que esa elección fue uno de los factores que condujeron al éxito del resultado final (Glendon, 2011: 208). Cuando Malik presentó el borrador para su votación en la Asamblea General, reconocería al chileno Hernán Santa Cruz «por hacer presente la gran visión humanista del mundo latinoamericano» (Glendon; 2011: 354). 
El 9 de diciembre de 1948, concluidos los trabajos de redacción, y faltando solo un día para la adopción de la Declaración Universal de Derechos Humanos en el Tercer Período de Sesiones de la Asamblea General de la ONU, Hernán Santa Cruz haría uso de la palabra para expresar, con enorme solemnidad y emoción, que:

En pocas horas más, las 58 naciones aquí representadas promulgarán la primera declaración internacional de derechos del hombre. Tal acto, por su trascendencia, justificará por sí solo la Tercera Sesión de la Asamblea General de las Naciones Unidas. De ahora en adelante, todos los seres humanos sabrán en qué consisten la dignidad y los derechos que tienen en igualdad, desde su nacimiento. ${ }^{19}$

Finalmente, el 10 de diciembre de 1948, en París, se vivía un momento que Santa Cruz describió posteriormente como:

Verdaderamente histórico, en el que se había conseguido un consenso sobre el supremo valor de la persona humana; un valor que no nacía de la decisión de los poderosos, sino más bien era la existencia la que nos daba el inalienable derecho a vivir libres para querer y libres de la opresión y para desarrollar la propia personalidad [...] había una atmósfera de genuina solidaridad y fraternidad entre los hombres y mujeres de todas las latitudes, algo que no he vuelto a ver en otras reuniones internacionales (Glendon, 2011: 248).

Esa noche, con 48 votos a favor, 8 abstenciones, 2 ausentes y o votos en contra, se aprobó la Declaración Universal de Derechos Humanos, iniciándose una nueva etapa para el Derecho Internacional para los derechos humanos y para la humanidad.

\section{Conclusión}

El nacimiento de la Declaración Universal de Derechos Humanos y el reconocimiento de los derechos contenidos en ella, se explican a partir de la confluencia de circunstancias históricas, corrientes de pensamiento y genios personales. Sin embargo, entre todos aquellos elementos apreciables, destaca nítidamente la importancia de las ideas presentadas por los representantes diplomáticos de América Latina en general, y defendidas por el chileno Hernán Santa Cruz en particular, quienes acumulaban experiencia negociadora a partir de los borradores preparados para configurar la Declaración Americana de Derechos y Deberes del Hombre.

En el caso de Santa Cruz, cabe subrayar que jugó un papel determinante en la redacción y aprobación de la Declaración Universal de Derechos Humanos, tanto con sus aportes sustantivos al texto como a través del liderazgo político asumido con res-

19. Para más información, véase «Hernán Santa Cruz», canal Ricardo gfn en Youtube, 25 de abril de 2019, disponible en https://youtu.be/vKpqloFsRUo. 
pecto al bloque latinoamericano y los puentes tendidos con representantes de otras regiones del mundo. Estos elementos, unidos a su rol posterior en la formulación del Pacto Internacional de Derechos Económicos, Sociales y Culturales, donde se le atribuye, entre otros, la autoría del artículo 11.1 sobre el derecho a la alimentación (Infante, 2018: 508), su liderazgo en el reconocimiento del derecho a la autodeterminación de los pueblos y su labor como funcionario internacional contra la discriminación racial, llevan a concluir que Hernán Santa Cruz fue un diplomático al servicio de los derechos humanos y una figura clave en la configuración del derecho internacional de los derechos humanos.

El momento histórico del fin de la Segunda Guerra Mundial y la confluencia de los intereses internacionales de los gobiernos latinoamericanos explican, en parte, la favorable coyuntura que supo aprovechar Hernán Santa Cruz para plasmar, en la Declaración Universal de Derechos Humanos, los conceptos que representaban parte de su ideario político y social, representativo del pensamiento de una generación latinoamericana sensible a las problemáticas sociales tan dramáticamente evidenciadas durante el siglo XX y también, lamentablemente, en el siglo XXI.

Nunca sabremos cuál hubiera sido el resultado final de la negociación de la Declaración Universal de Derechos Humanos sin el ímpetu diplomático del representante chileno, pero la realidad indica que Hernán Santa Cruz asumió con éxito un liderazgo que permitió el reconocimiento universal de los derechos humanos, levantando las banderas de su indivisibilidad e interdependencia.

\section{Referencias}

Allende, Salvador (1939). La realidad médico-social chilena. Disponible en https:// bit.ly/3JmmGFK.

Bernstein, Enrique (1984). Recuerdos de un diplomático: Haciendo camino 1933-1957. Volumen 1. Santiago: Andrés Bello

Glendon, Mary Ann (2011). Un mundo nuevo: Eleanor Roosevelt y la Declaración Universal de Derechos Humanos. Ciudad de México: Fondo de Cultura Económica. Humphrey, John P. (1984). Human rights and the United Nations: A Great Adventure. Nueva York: Transnational Publishing.

InfANTE, María Teresa (2018). «El rol del derecho internacional en las instancias jurídicas multilaterales». En Juan Somavía y Pedro Oyarce Chile, Actor del sistema multilateral: Una tradición nacional (pp. 481-516). Santiago: Academia Diplomática Andrés Bello.

MANCISIDOR, Mikel (2017). «El derecho humano a la ciencia: Un viejo derecho con un gran futuro». Anuario de Derechos Humanos, 13: 211-221.

Morsink, Johannes (1999). The universal declaration of human rights: Origins, drafting and intent. Philadelphia: University of Pennsylvania Press. 
RojAs, Germán (2018). «La Organización de las Naciones Unidas para la Alimentación y la Agricultura (FAO)». En Juan Somavía y Pedro Oyarce, Chile actor del sistema multilateral: Una tradición nacional (pp. 395-413). Santiago: Academia Diplomática Andrés Bello.

Ross, César (2014). «Hernán Santa Cruz: Del pensamiento a la acción». Horizontes Latinoamericanos, 2 (1): 79-92.

Santa Cruz, Hernán (1945). Vinculación intelectual entre Brasil y Chile. Santiago: Instituto Chileno Brasileño de Cultura.

-. (1984). Cooperar o perecer: El dilema de la comunidad mundial. Tomo 1. Buenos Aires: GEL.

-. (1995). «La creación de las Naciones Unidas y de la Cepal». Revista de la Cepal, 57.

-. (1999). «El porqué de mi incorporación a la vida internacional». Revista Diplomacia, 78.

Schabas, William (2013). The universal declaration of human rights: The travaux préparatoires. Cambridge: University Press.

Toro, Sergio (2018). «Chile en el ECOSOC». En Juan Somavía y Pedro Oyarce (editores), Chile actor del sistema multilateral: Una tradición nacional (pp 245-281). Santiago: Academia Diplomática Andrés Bello.

UlloA, Erna y Cristian Medina (2019). «Outline of a diplomatic leader in the international community». Human Rights Quarterly, 41 (4): 962-981.

\section{Agradecimientos}

El autor agradece especialmente el impulso y los valiosos comentarios del profesor Mikel Mancisidor.

\section{Sobre el autor}

Felipe Aravena es diplomático y ha sido cónsul de Chile en Ecuador, representante alterno de Chile ante la OEA y presidente de la Comisión de Asuntos Migratorios de la OEA. Estudió Derecho en la Universidad de Chile, Ciencia Política en la Universidad Católica y Diplomacia en la Academia Diplomática Andrés Bello, todas estas instituciones del mismo país. Fue becario del Instituto del Servicio Exterior de la Nación en Buenos Aires, Argentina, y del Clingendael Institute of International Relations en La Haya, Países Bajos. Es Master of Laws en International Human Rights and Humanitarian Law por American University, Washington College of Law. Su correo electrónico es fa8727a@student.american.edu. (D) http://orcid.org/oooo-0002-9450-4108. 